\title{
Making menus musical
}

\author{
Stephen A. Brewster and Murray G. Crease \\ Department of Computing Science \\ University of Glasgow, Glasgow, G12 8QQ, UK. \\ Tel: +44 (0)141330 4966 / Fax: +44 (0)141330 4913 \\ stephen@dcs.gla.ac.uk or murray@gtnet.com \\ www.dcs.gla.ac.uk/ stephen/
}

\begin{abstract}
Future human-computer interfaces will use more than just graphical output to display information. In this paper we suggest that sound and graphics together can be used to improve interaction. We describe an experiment to improve the usability of standard graphical menus by the addition of sound. One common difficulty is slipping off a menu item by mistake when trying to select it. We designed and experimentally evaluated sonically-enhanced menus to) try and overcome this problem. The results from the experiment showed a significant reduction in the subjective effort required to use the new menus along with significantly reduced error recovery times. A significantly larger number of errors were also corrected with sound.
\end{abstract}

KEY WORDS Menus, audio, multi-modal widgets, earcons, sonification.

\section{INTRODUCTION}

Human-computer interfaces currently rely almost entirely on graphical feedback to present information to the user. However, research is now showing that sound combined with graphics can significantly improve usability by taking advantage our natural ability to share tasks across sensory modalities, for example Alty (1995), Beaudouin-Lafon \& Conversy (1996) and Brewster et al. (1995). Such multimodal interfaces allow a greater and more natural communication between the computer and the user. They also allow the user to employ appropriate sensory modalities to solve a problem, rather than just using one modality (usually vision) to solve all problems. We believe that for these reasons sound should become a standard, integrated component in human-computer interfaces of the future. ( )ne question that remains is how should sound be used to improve usability?

This paper describes an experiment to investigate the integration of sound into standard graphical menus to overcome usability problems. Menus are a standard feature of most graphical interfaces because they are a convenient way of grouping commands to improve usability. Norman (1991) has a detailed discussion of all aspects of menus and their use. The design of menus has become almost standard across systems. However, they are not without problems. One of these is the misselection of menu items due to inadequate feedback. Norman has very little discussion of the feedhack required to indicate a selection. He does say (p 157) "...the system should provide feedback to the users confirming selection and informing them of its progress". He does not suggest how this should he done. It is normally assumed that the visual highlighting of the menu item is sufficient. We believe that this is not the case.

\subsection{The problem with menus}

Pull-down menus are operated by pressing the mouse button down in the menu bar (or on hot-spot to bring up a pop-up menu). On some systems (e.g. the Macintosh) the mouse button must be held down so that the menu 
remains visible, on other systems (e.g. Windows95) the menu may stay pulled down until the user presses the mouse button again. This paper concentrates on the former. Moving the cursor along the menu bar highlights the menu title the cursor is in and displays the items in that menu. When the cursor is moved into the menu the items are highlighted as the mouse moves over them (unless the item is a divider or greyed-out). If the cursor is moved out of the menu the menu remains displayed but the highlight is removed. This provides a back-out facility in case the user decides that no menu item is to be chosen. If the cursor is over a valid item when the button is released that item is selected and the menu is removed. On some systems (e.g. the Macintosh) the user can set the item selected to flash between zero and three times to indicate that it has been selected. If the button is released when the cursor is not over a valid menu item, the menu is simply removed. For more details on menu interactions see Crease (1996).

A study of menu interactions showed three possible outcomes of a selection: A correct selection, an incorrect selection and no selection.

An incorrect selection could occur for one of two reasons: 1) The wrong menu item was mistakenly selected - a mis-selection. In this case the user simply chose the wrong item; 2) The cursor slipped onto the wrong menu item accidentally as the button was being released - an item slip. Our study of menus showed that, sometimes, just as the mouse button was released users could slip into an adjacent menu item. This happened partly because the action of releasing the mouse button could move the mouse a little and also because users often started to move the mouse to the location of the next action before the mouse button was released.

Similarly, no selection could happen for one of three reasons: 1) The user decided not to select anything and moved the cursor out of the menu before releasing the button: 2) The cursor slipped off the edge of the menu as the button was being released - a menu slip; 3) The user selected a disabled item or a divider.

Menu and item slips reduce the usability of menus and can sometimes cause more serious problems. For example, if the user slipped off when trying to save and did not notice, the data would not be saved with perhaps serious consequences. We decided to investigate these problems further to see if menus could be improved.

\subsection{Action Slips and Closure}

Why do item and menu slips occur? As mentioned, they can occur because the release of the mouse button can move the mouse itself. However, the errors are also both examples of action slips (Reason, 1990). These errors occur with expert users who perform many simple operations (such as button clicks and menu selections) automatically and do not explicitly monitor the feedback from each interaction. Lee (1992) describes such errors thus (p 73): “...as a skill develops, performance shifts from 'closed loop' control to 'open-loop' control, or from monitored mode to an automatic, unmonitored mode of processing." As users become familiar with a simple task they no longer monitor the feedback from it so closely. In this case the automatic task is the menu selection (which most users are very familiar with). The user will be concentrating more on the main task he/she is trying to perform than on the menu selection.

One problem that exacerbates the difficulties of actions slips is closure (Dix et al., 1993). Closure occurs when a user perceives a task as being completed. In some cases the task may appear to be completed when it is not. The user may experience closure and carry on to do something else and cause an error. Brewster $e t$ al. (1995) and Dix \& Brewster (1994) investigated such a situation that could occur with graphical buttons. In this case, users could slip off a button by mistake and not press it. This happened because as the graphical button highlighted (when the mouse button was pressed), users experienced closure and carried on to their next task. In fact the graphical button was not actually pressed until the mouse button was released. After analysis, Brewster et al. suggested three criteria necessary to make action slips and closure a problem. In terms of menus these would be:

i) The user reaches closure after the cursor is moved into the desired menu item and the text is highlighted.

ii) The visual focus of the next action is at some distance from the target menu item.

iii) The cursor is required at the new focus.

In this case, when the desired menu item has been highlighted the user experiences closure. The task is, in reality, not completed until the mouse button is released over the desired menu item. However, because of closure the user may begin to move on to his/her next task before the menu selection has been completed. If this 
task requires the cursor and visual focus to be away from the menu, all the conditions for an action slip have been met.

An example of this error consider selecting 'Save' from the file menu in a word processor. The user will be more concerned with the document being saved than the mechanics of the menu, making action slips likely. He/she would be typing in the document and decide to save. The mouse (and visual attention) would be moved up to the menu to select save, the menu item would be highlighted, the user would see the highlight, reach closure and then begin to move back to the document to continue typing. If this return movement is done 100 soon, the mouse may slip on to another item or out of the menu before the interaction is complete. In many systems no feedback is given to indicate a save has been performed, so the user would have no indication of his/her error.

\subsection{Overcoming the Problems}

In order to solve the problems of action slips and accidental mouse movements users must perceive the feedback from the menu. Therefore the right feedback must be given to ensure users know what is going on (Reason, 1990). The Macintosh-style multiple flash goes some way to dealing with this problem, but is not a complete solution because any feedback from the menu requires the user's visual attention to be on the menu and if the second criterion above is met, this will not be the case. In this paper we suggest using auditory feedback to solve the problems. Why use sound, why not just use extra graphical feedback? It is difficult to solve these problems with extra graphics. Graphics displayed on the menu will not be seen by users because their attention will have moved back to the main task they engaged in. The visual system has a narrow area of focus which means that users cannot look at the menu as well as their main task (Brewster, 1994). Information could be displayed at the mouse location but we do not know if users are looking there either. In this case, non-speech sound has certain advantages. It can be heard from all around, it is good at getting our attention whilst we are looking at something else and it does not disrupt our visual attention. It is also language independent. If we give menu information in sound then we do not need to know where users are looking. If users must look at the menu then it forces them to stop what they are doing for their main task and causes the interface to intrude upon the task they are trying to perform, sound does not have such drawbacks.

\subsection{Previous Work in the Area}

As mentioned above, Brewster et al. (1995) investigated the use of sound to improve interaction with graphical buttons. Buttons have a subset of the problems of menus because users can slip off a button in a similar way to a menu. To solve the problems sounds were added for when the mouse button was pressed down in a graphical button and when a correct selection was made. The results of adding sound to buttons significantly improved their usability. Error recovery was significantly faster and required fewer mouse clicks than standard buttons. Participants also significantly preferred them to standard graphical buttons. The knowledge gained from that experiment suggested that we might be able to overcome some of the problems of menus in the same way.

Other work on sonifying widgets, for example scrollbars Beaudouin-Lafon \& Conversy (1996) and Brewster et al. (1994), have proved successful in improving usability. We therefore believed that sound would allow use to improve the usability of menus.

Much work has been done on the optimal layout and design for menu structures (see Norman for a full review) but little has been done 10 investigate the selection of items from menus. A notable exception is the research by Walker et al. (1991) who proposed some design improvements for graphical menus to increase their usability hy decreasing the time taken to move the cursor to the desired item. Their two main approaches were: The use of impermeable borders around menus and to increase the size of items further away from the start point in the menu to make them easier to hit. It was found that the use of impermeable borders (ones that the mouse could not move through) reduced the time taken to make a selection. The user could move the mouse to the top, bottom or right edge of the menu and not be able to move through. Therefore, the user could aim to overshoot the menu item at the edge where there was an impermeable border knowing that it was not possible to do so. This eliminated the time taken to make the secondary movement required to correct the initial overshoot. The user could leave the menu without making a selection by moving the mouse out the left edge of the menu. One problem was that it did make the 
menus less flexible because users had to leave them from the left side only.

Walker $e$ t al.'s other solution was to increase the point size of items as their distance from the top of the menu increased, i.e. increasing the size of the target area. This allowed the user to make a movement towards the target more quickly. It did however also make the distance to the target greater. If the menu became ton long, two movements would have to be made to reach the target, thus eliminating any benefits gained.

There has been some previous work on adding sound to menus, although to solve different problems. Karshmer et al. (1994) added sounds to a menu system to aid navigation for blind users. He was not trying to overcome the slip off problems but to aid users in navigating around menus structures. The navigation was implemented by either changing the tones and timbres of the items in the menus, or by using synthetic speech to tell users their position in the hierarchy. Navigation was not the focus of our work here, our aim was to overcome slip off problems in graphical menus for sighted users.

\section{EXPERIMENT}

As there had been no other work to try and overcome menu selection errors with sound we decided to design an experiment to discover if auditory feedback could improve usability. For the experiment we needed to be able to differentiate three types of errors: An item slip, a menu slip and a slip onto a divider or disabled item. We could easily differentiate the latter two (because we knew where the participants were supposed to be clicking) but the former was more difficult. How could we tell if users slipped off a menu item or were really choosing the one on which they released the mouse?

We conducted an initial investigation into menu interactions to investigate ways in which we might be able to find out if an item slip had occurred. We noticed that when users selected an item correctly they stayed on the item longer than when an item slip occurred. We therefore decided to use timing information to help us identify correct selections and item slips. In a pre-test we added a timer to a simple menu system so that we could investigate how long it took users to select items (Crease, 1996). From this we discovered that for a correct selection users held the mouse over the item for an average of $265 \mathrm{msec}$ before releasing. When an item slip was made the cursor was over the item for just 17 msec. For correct selections users moved the mouse to the item and did some cognitive processing to ensure it was the correct menu item before releasing the mouse button. For an item slip there was no cognitive processing, just the mouse button release time. This gave us a method for identifying correct selections and item slips.

\subsection{Experimental Hypotheses}

The extra feedback heard by participants should make the task easier because they will be able to tell they have made errors and recover from them more readily. This should result in an overall reduction in subjective workload and in particular a reduction in the effort required for the lask. There should be no increase in annoyance due to the sounds as they will be providing information that the participants need.

The time taken to recover from item and menu slips should be reduced because users will know that they have made errors more quickly. The number of errors corrected by the participants (i.e. corrected before the error dialogue comes up to say that an error has been made) should also be increased as the demanding auditory feedback will be noticed.

\subsection{Main Task}

Figure 1 shows the interface to the task the participants had to perform. It was based on a car parts ordering system. Participants had to send car parts to destinations using a menu system. In the figure the Parts and Destinations menus are shown. At the bottom of the screen the boxes with 'fan belt' and 'Auchterarder' in them show the participants the part and destination they must choose. Once a part and destination pair had been chosen, the participants had to select 'Accept' on the File menu. This terminated the interaction. If one or both of the chosen items was incorrect an error dialogue was displayed and corrections had to be made before participants could continue. If an item or menu slip occurred on any of the Parts, Destinations or File menus then the participant would not be able to carry on to the next pair until they had noticed and corrected the error.

The task was designed to be simple so that the participants could easily learn it and reach a level of automaticity in the task where slip-off errors would occur. 


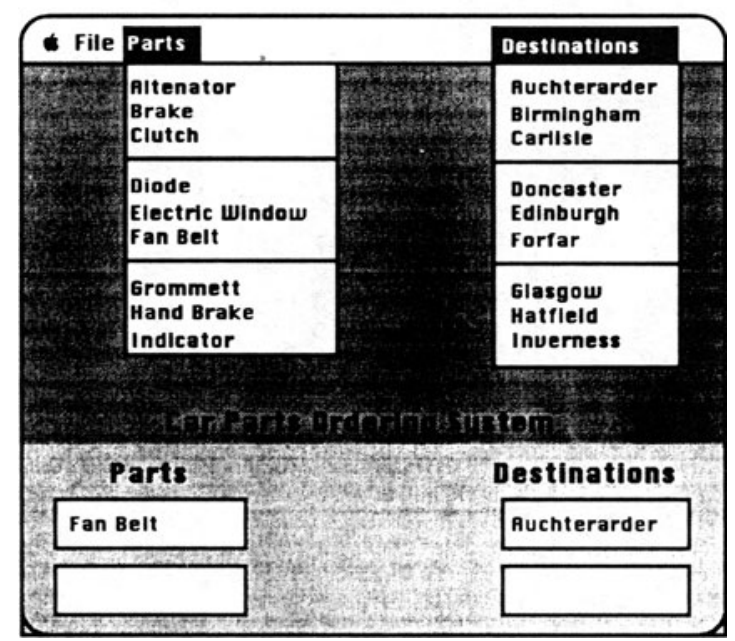

Figure 1: The screen of the menu testing program. The parts and destinations menus are shown.

\subsection{Sounds}

The sounds used were based around structured audio messages called Earcons (Blattner et al., 1989, Brewster et al., 1993). Earcons are abstract, musical tones that can be used in structured combinations to create sound messages to represent parts of an interface. The sounds were created using the earcon guidelines proposed by Brewster et al. (1995).

Three earcons were needed to deal with the problems described above:

1. An earcon was played when a menu was displayed. We used a family of organ sounds to indicate that the menus were related (part of the same application). The File menu had a percussive organ, the Parts menu a drawbar organ and the Destinations menu a rock organ. A low intensity, continuous note at pitch $C_{3}(523 \mathrm{~Hz})$ was played for each of the menus. The sound continued as long as the cursor was in the menu. If the user moved the cursor out of the menu the sound stopped. The method proved effective in the design of sonicallyenhanced buttons (Brewster et al., 1995) so was used again here.

2. To deal with item slips a combination of two earcons was used. A highlight sound was created that was similar to the standard graphical highlight. This was again a continuous, low intensity tone which used the timbre of the menu that the cursor was in. The sound alternated in pitch from $\mathrm{B}_{2}(987 \mathrm{~Hz})$ for odd numbered items and $\mathrm{E}_{3}(329 \mathrm{~Hz})$ for even numbered items. So the first item in the menu would be played at $E_{3}$, the second at $B_{2}$ and the third at $E_{3}$. This sound started when the mouse had been over an item for half a second. Only two sounds were needed to indicate the movement from one menu item to another. These pitches were chosen to make the two earcons sound distinct. This sound was stopped if the user moved the mouse out of the menu or moved over a divider or disabled item.

3. The final earcon indicated a selection. This could be either a correct selection or an item slip. For a correct selection (in a menu item for longer than $17 \mathrm{msec}$ ) the earcon was based around the timbre of the menu the cursor was in and the pitch of the sound was based on the highlight sound for the menu item. Using these as a base, two $40 \mathrm{msec}$ duration tones were played at a higher intensity. To indicate an incorrect selection the timbre of the current menu was again used. However, this time a fixed rhythm of three notes of $40 \mathrm{msec}$ each at pitch $\mathrm{C}_{2}$, $\mathrm{B}_{2}$ then $\mathrm{F}_{2}$ was played (these sounded discordant and therefore attention-grabbing). This was not dependent on the highlight sound for the current menu item. This sound was always the same, indicating the item slip error in each of the different menus. If the user released the mouse over a divider then no sound was played.

To avoid any potential annoyance due to the earcons we made sure they were all played at low volume. The menu earcon (1) indicated a menu slip by a lack of sound and the highlight earcon (2) didn't start playing until the participant had been over an item for half a second. In a normal, fast interaction this sound was not played, the user only heard the menu sound (1) and the selection sound (3).

\subsection{Participants}

Twelve participants were used. They were undergraduate and postgraduate students from the Department of Computing Science at the University of Glasgow. All were experts with more than three years experience of graphical interfaces and menus.

\subsection{Experimental Design and Procedure}

The experiment was a two-condition, repeatedmeasures within-subjects design. The order of presentation was counterbalanced to avoid any learning effects across conditions. One group of six participants performed the auditory menu condition first and the 
other used standard visual menus first. Ten minutes of training was given before each condition. During each condition the participants had to enter 150 parts to destinations pairs as quickly as possible. Instructions were read from a prepared script.

To get a full measurement of usability we used a range of quantitative and qualitative measures. These were error rates, error recovery times and workload (Hart \& Staveland, 1988).

Hart \& Staveland (1988) break workload into six different factors: Mental demand, physical demand, time pressure, effort expended, performance level achieved and frustration experienced. We used the NASA-Task Load Index (TLX) for estimating workload. To this we added a seventh factor: Annoyance. One of the main concerns of potential users of auditory interfaces is annoyance due to sound pollution. This is often given as a reason for not using sound at the human-computer interface. In the experiment described here the annoyance due to auditory feedhack was measured to find out if it was indeed a problem. We also asked our participants to indicate overall preference, i.e. which of the two interfaces they felt made the task easiest. Participants had to fill in workload charts after both conditions of the experiment.

\section{RESULTS}

\subsection{Workload Results}

Figure 2 shows the average workload score for each category. They were scored in the range 0-20. The average workload (based on the six standard workload factors: Mental, physical, time, effort, frustration and performance) was 10.02 for the auditory condition and 11.04 for the visual. This difference was significant $\left(\mathrm{T}_{11}=4.42, \mathrm{p}=0.007\right)$ indicating that the participants found the task easier with the sonically-enhanced menus. This confirmed the hypothesis.

Paired T-tests were carried out on the auditory versus visual conditions for each of the workload categories. Average effort was reduced significantly from 13.9 in the visual condition to 12.5 in the auditory condition $\left(\mathrm{T}_{11}=2.43, \mathrm{p}=0.03\right)$ confirming the hypothesis.

There was no significant difference in terms of annoyance $\left(\mathrm{T}_{11}=1.43, \mathrm{p}=0.18\right)$. Six of the participants rated the visual condition more annoying, five the auditory condition more annoying and one rated them

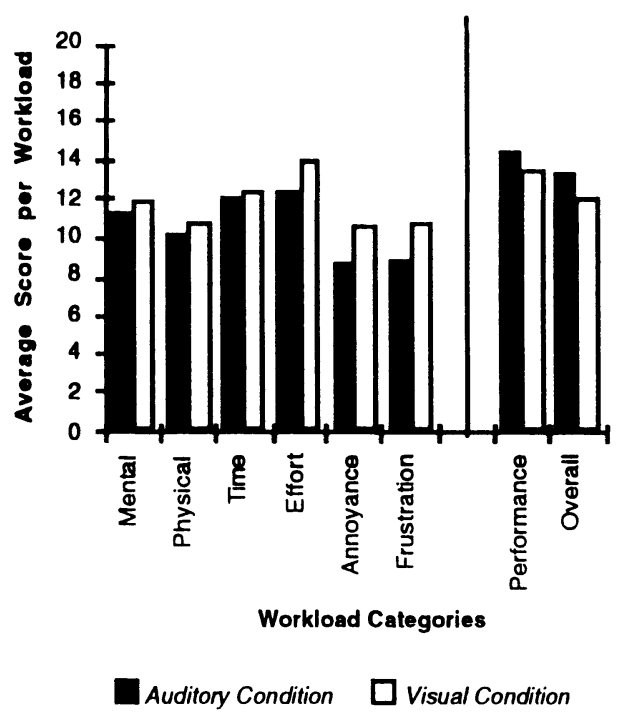

Figure 2: Average workload scores for the two conditions. In the first six categories higher scores mean higher workload. The final two categories, performance and overall preference, are separated because higher scores mean less workload.

the same. This indicated that the participants did not find the auditory feedback to be annoying confirming the hypothesis.

\subsection{Time and Error Results}

The overall number of errors corrected (i.e. before the error dialngue appeared) increased significantly from $55 \%$ to $92 \%$ in the auditory condition $\left(\mathrm{T}_{11}=3.982\right.$, $\mathrm{p}=0.002$ ), confirming the hypothesis. The percentage of item slips corrected increased significantly to $70 \%$ in the auditory condition from $17 \%$ in the visual $\left(\mathrm{T}_{11}=4.21\right.$, $\mathrm{p}=0.001$, again confirming the hypothesis. The percentage of menu slips corrected increased to $83 \%$ in the auditory condition from $63 \%$ in the visual. This was not a significant improvement $\left(\mathrm{T}_{11}=1.11, \mathrm{p}=0.289\right)$. Two participants made no menu slips at all in the auditory condition.

The average time taken to recover (detection and correction of the error) from all errors decreased significantly in the auditory condition ( 2.38 secs) compared to the visual $(4.18$ secs $)\left(T_{11}=4.04\right.$, $\mathrm{p}=0.0009$ ). Figure 3 shows the average time each participant took to recover from an error in each condition (both item and menu slip errors are included). 


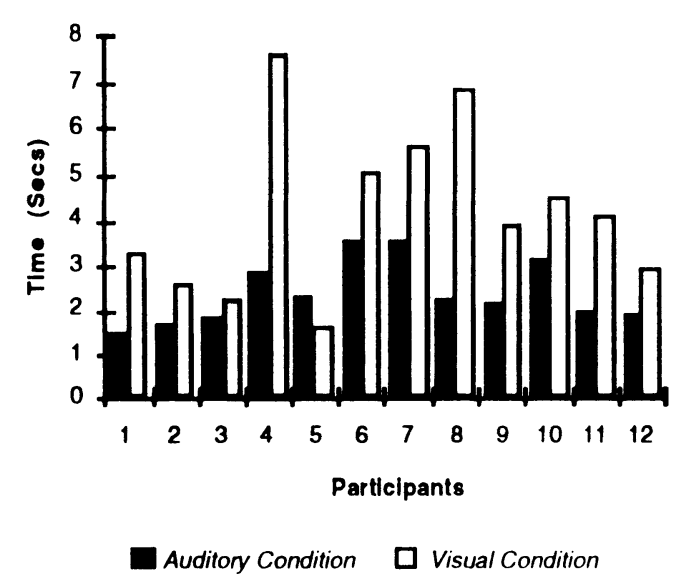

Figure 3: Average error recovery times for all errors.

The average time taken to recover from an item slip decreased significantly from 4.1 secs in the visual condition to 2.53 secs in the auditory $\left(\mathrm{T}_{11}=2.55\right.$, $\mathrm{p}=0.03$ ). The recovery time from a menu slip decreased from an average of 3.07 secs in the visual condition 10 1.57 secs in the auditory. A wide variance in the times in the visual condition meant that an F-Test was conducted. This showed a significant reduction in the variance in the auditory condition $\left(\mathrm{F}_{11}=18.11, \mathrm{p}=1.76 \times 10^{-5}\right)$. The range of times decreased from a minimum of 1.87 secs and a maximum of 11.25 secs in the visual condition 10 between 1.73 secs and 2.08 secs.

\subsection{Discussion}

The workload analysis showed that the overall workload, and in particular effort expended, was reduced significantly when using the sonically-enhanced menus. It was reduced because the sonic enhancements meant that participants needed to expend less effort to notice and recover from menu and item slips. This was not at the expense of making the menus more annoying to use. This, along with previous results from sonifying graphical widgets indicates that sound can provide a significant qualitative improvement in a user's experience with a system.

The results also showed that the integration of sound allowed faster recovery from errors. Both menu and item slip error recovery rates showed a significant improvement in the auditory condition. Participants could tell they had made an error and correct it significantly faster than in the visual condition. The menu slip off results showed that the earcons reduced the variance in the time taken to recover from an error. The data indicated that the better performers stayed at a similar standard, whilst the poorer performers improved greatly.

The reason the sonic feedback improved error recovery rates was that the participants' visual focus moved to the next menu before the previous selection was completed. In the visual condition the avoidable menu-selection feedback was missed, whereas in the auditory condition the demanding feedback ensured the user was alerted.

Although the item slip recovery rate was significantly improved, the item slip sound was occasionally played incorrectly. This happened if a participant interacted with the system very slowly and their items slips took longer than $17 \mathrm{msec}$. These false positives occurred as some users moved the mouse around much more slowly than others. To solve this problem a future the system could include a control panel, similar to the one that allows a user to set their preferred double-click speed. which would allow a user to set their preferred menu selection speed.

\section{FUTURE WORK}

This work is part of an overall project to develop a sonically-enhanced interface toolkit (Brewster, 1995). The toolkit will consist of all of the standard widgets but they will be enhanced with sound to improve their usability.

One interesting area of future work would be to investigate the use of automatic error correction. Our pre-test showed that we could identify item slips by the time they took. This means that we could add an auto correction feature to the menus so that if a selection took place that the system identified as an item slip then it could be rejected and the adjacent item where the mouse had been for a longer amount of time could be selected. This would avoid errors of this type occurring. We will investigate the use of sound together with automatic error correction in a future experiment.

\section{CONCLUSIONS}

This work has demonstrated the power of multimodal interactions. By combining the advantages of the visual and auditory senses, problems of interacting with menus 
can be overcome. The results have shown that integrating earcons into graphical menus can reduce the subjective workload required to use them. It can also increase the number of errors corrected and reduce the time taken to recover from errors. These advantages were gained without making the menus more annoying to use.

Using sound in this way means that users can concentrate on their main task without the interface intruding. The combination of graphics and sound provided a significant qualitative and quantitative improvement in the participants' experience with the menus. Future interface designers can use sound in this way and know that they will he improving the usability of their systems.

\section{REFERENCES}

All of the references by Brewster along with sounds samples are available from http:/l www.dcs.gla.ac.uk/ stephen/

Alty, I.L. (1995). Can we use music in humancomputer interaction? In Kirby, Dix \& Finlay (Eds.), Proceedings of People and Computers X, HCl'95, (pp. 409-423), Huddersfield, UK: Cambridge Iniversity Press.

Beaudouin-Lafon, M. \& Conversy, S. (1996). Auditory illusions for audio feedback. In Tauber (Ed.), CHI'96 Conference Companion, (pp. 299-300), Vancouver, Canada: ACM Press, Addison-Wesley.

Blattner, M., Sumikawa, D. \& Greenberg, R. (1989). Earcons and icons: Their structure and common design principles. Human Computer Interaction, 4, 11-44.

Brewster, S.A. (1994) Providing a structured method for integrating non-speech audio into human-computer interfaces. PhD Thesis, Iniversity of York..

Brewster, S.A. (1995). The development of a sonically-enhanced widget set. In Blumenthal. Gornostaev \& Unger (Eds.). Proceedings of EWHCI'95. (pp. 126-129), Moscow, Russia: International Centre for Scientific and Technical Information.

Brewster, S.A., Wright, P.C., Dix, A.J. \& Edwards, A.D.N. (1995). The sonic enhancement of graphical buttons. In Nordby, Helmersen, Gilmore \& Arnesen (Eds.), Proceedings of Interact'95, (pp. 43-48), Lillehammer, Norway: Chapman \& Hall.
Brewster, S.A., Wright, P.C. \& Edwards, A.D.N. (1993). An evaluation of earcons for use in auditory human-computer interfaces. In Ashlund, Mullet, Henderson, Hollnagel \& White (Eds.), Proceedings of INTERCHI'93, (pp. 222-227), Amsterdam: ACM Press, Addison-Wesley.

Brewster, S.A., Wright, P.C. \& Edwards, A.D.N. (1994). The design and evaluation of an auditoryenhanced scrollbar. In Adelson, Dumais \& Olson (Ed.), Proceedings of CHI'94, (pp. 173-179), Boston, Ma.: ACM Press, Addison-Wesley.

Brewster, S.A., Wright, P.C. \& Edwards, A.D.N. (1995). Experimentally derived guidelines for the creation of earcons. In Kirby, Dix \& Finlay (Eds.). Adjunct Proceedings of HCI'95, (pp. 155-159), Huddersfield, UK

Crease, M. (1996) Making Menus Musical. Undergraduate Thesis, University of Glasgow.

Dix, A., Finlay, J., Abowd, G. \& Beale, R. (1993). Human-Computer Interaction. London: Prentice-Hall.

Dix, A.J. \& Brewster, S.A. (1994). Causing trouble with buttons. In Ancillary Proceedings of HCI'94. Sterling, UK: Cambridge University Press.

Hart, S. \& Staveland, L. (1988). Development of NASA-TLX (Task Load Index): Results of empirical and theoretical research., In Hancock \& Meshkati, Eds.. Human mental workload. Amsterdam: North Holland B.V.

Karshmer, A., Brawner, P. \& Reiswig, (i. (1994). An experimental sound-based hierarchical menu navigation system for visually handicapped use of graphical user interfaces. In Proceedings of ACM ASSETS 94 , (pp. 123128), Marina Del Rey, CA, USA: ACM Press.

Lee, W.O. (1992). The effects of skill development and feedback on action slips. In Monk, Diaper \& Ilarrison (Eds.), Proceedings of $\mathrm{HCl}^{\prime} 92$, (pp. 73-86), York. IJK: Cambridge University Press.

Norman. K. (1991). The psychology of menu selection. Norwood, NI, USA: Ablex Publishing.

Reason, J. (1990). Human Error. Cambridge, UK: Cambridge University Press.

Walker, N., Smelcer, J. \& Nilsen, E. (1991). Optimizing speed and accuracy of menu selection: $\Lambda$ comparison of walking and pull-down menus. International Journal of Human-Computer Studies, 35, 871-89). 